\title{
Antimicrobial Activity of Carbon Nanoparticles Isolated from Natural Sources against Pathogenic Gram-Negative and Gram-Positive Bacteria
}

\author{
Sheena Varghese, ${ }^{1,2}$ Sunny Kuriakose, ${ }^{2}$ and Soumya Jose ${ }^{1,2}$ \\ ${ }^{1}$ Research and Development Centre, Bharathiar University, Coimbatore 641046, India \\ ${ }^{2}$ Research and Postgraduate Department of Chemistry, St. Thomas College, Mahatma Gandhi University, Palai, Kerala 686574, India \\ Correspondence should be addressed to Sunny Kuriakose; skresearchgroup@rediffmail.com
}

Received 9 April 2013; Accepted 24 May 2013

Academic Editor: Mingwang Shao

Copyright (C) 2013 Sheena Varghese et al. This is an open access article distributed under the Creative Commons Attribution License, which permits unrestricted use, distribution, and reproduction in any medium, provided the original work is properly cited.

\begin{abstract}
This paper describes the isolation of carbon nanoparticles (CNPs) from kitchen soot, characterization of the CNPs by UV/visible spectroscopy, SEM and XRD, and their antimicrobial action. The antibacterial activity of the isolated carbon nanoparticles was tested against various pathogenic bacterial strains such as Gram-negative Proteus refrigere and Pseudomonas aeruginosa and Grampositive Staphylococcus aureus and Streptococcus haemolyticus. The inhibition zones were measured, and it was found that the carbon nanoparticles isolated from natural sources are active against these Gram-negative and Gram-positive bacterial strains.
\end{abstract}

\section{Introduction}

Carbon is one of the most abundant elements in nature. Carbonbased nanomaterials have attracted great interest in recent decades. A broad range of carbon nanostructures have been prepared, such as carbon nanotubes, fullerenes, nanofibers, nanodiamond, carbon nanoonions, and other carbonaceous nanomaterials. Carbon nanoparticles have excellent applications because of their unique chemical and physical properties. CNPs can result in a reduction in material weight and in the obtaining of materials with higher impact strength, high surface area per unit volume, electrical conductivity, optical properties, thermal stability, flame resistance, and dimensional stability $[1,2]$.

Potential newly found application of carbon nanoparticles occurs in nanoscience nanotechnology in such diverse areas as protective coatings, antimicrobial agents, electronic and optical devices, microsensors, and pollution prevention materials. In biological science carbon based nanomaterials have been utilised as excellent platforms for facilitating biochemical reactions and processes, such as sensitive recognition of antibodies, sequencing of nucleic acids, bioseparation, and biocatalysis $[3,4]$.
Due to the emergence of infectious diseases caused by different pathogenic microbes the pharmaceutical companies and the researchers are searching for new antimicrobial agents. Some of antimicrobial agents are extremely irritant and toxic to humans. Thus, the formulation of new effective, resistance-free, low-cost, and natural origin antimicrobial agents is of great interest [4-9]. An antibacterial drug in medication is used to treat bacterial infections. In the present scenario nanoscale materials such as CNPs have emerged up as novel antimicrobial agents owing to their high surface area to volume ratio and its unique chemical and physical properties $[10,11]$. The integration of nanotechnology and microbiology leads to possible advance in the formulation of new types of antibacterial agents.

Carbon based nano materials have high antimicrobial activity. It has been established that single-walled CNTs exhibit a strong antimicrobial activity and can pierce bacterial cell walls [12-16]. As the size of carbon particles decreases down to nanoscale range their antimicrobial activity increases because of their larger surface area per unit volume and high proportion of atoms in the surface and near surface layers. In this work, we adopted a simple lowcost method for the isolation of carbon nanoparticles which 
exhibit strong antibacterial activity. On the basis of this enhanced effectiveness, carbonaceous nanomaterials were aptly employed to fight to human pathogens. The paper covers the isolation of carbon nanoparticles from natural sources and the analysis of the antimicrobial activity of these nanoparticles against selected bacterial strains.

\section{Materials and Methods}

\subsection{Isolation of Carbon Nanoparticles from Natural Sources}

2.1.1. Method of Collection. Carbon nanoparticles were isolated from kitchen soot. It is one of the inexpensive, versatile, and reproducible methods for the isolation of carbon nanoparticles. Soot is a general term that refers to impure carbon particles resulting from the incomplete combustion of a hydrocarbon. Natural woods such as branches of trees, and coconut husk are used for burning purpose. Glass plates of uniform sizes were hung above the hearth in a chimney so that smoke from this hearth will stick on the glass plate. The glass plates were labelled as day 1 , day 2, and so forth, and the corresponding glass plates were removed from the smoke hood after one day, two days and so forth up to seven days. The particles sticking on the glass plates were scratched off and collected. The soot after six-month duration was also collected and it was labeled as sample "infinity". The collected carbon particles were stored for further use by wrapping in aluminium foil without exposure to sunlight.

2.1.2. Purification. The crude kitchen soot was dissolved in acetone-water $(3: 1)$ mixture. The undissolved residue was discarded after centrifugation. The clear solution was collected and the solvent was evaporated off on a vacuum rotary evaporator, and the fluorescing CNP in the powder form was collected. The solubility of the nanoparticles was tested and was found to be soluble in acetone, methanol, chloroform, water, DMF, and DMSO.

2.2. Characterisation of Carbon Nanoparticle. The characterization techniques included UV/visible spectroscopy, Scanning Electron Microscopy (SEM), and X-ray Diffraction (XRD) $[17,18]$.

2.3. Preparation of Culture Media for Incubation of Microorganisms. $1.3 \mathrm{~g}$ nutrient broth powder was dissolved in $100 \mathrm{~mL}$ water by gently boiling in a $250 \mathrm{~mL}$ conical flask stoppered by cotton plug. The broth was sterilized by autoclaving at $15 \mathrm{lbs}$ pressure at $121^{\circ} \mathrm{C}$ for 15 minutes. It was cooled in a laminar hood, which was disinfected by cleaning thoroughly with absolute alcohol followed by UV irradiation for 20 minutes. A disinfected wire loop size stock microorganisms was transferred into the cold medium under laminar flow. $7.6 \mathrm{~g}$ Miller Hinton Agar (M. H. Agar) and 1.2 g Agar Agar were dissolved in $200 \mathrm{~mL}$ water in a capped bottle. The agar solution was sterilized by autoclaving at $15 \mathrm{lbs}$ pressure at $121^{\circ} \mathrm{C}$ for 15 minutes. Then it was cooled to $40-45^{\circ} \mathrm{C}$ in a laminar hood, which was disinfected by cleaning thoroughly with absolute alcohol followed by UV irradiation for 20

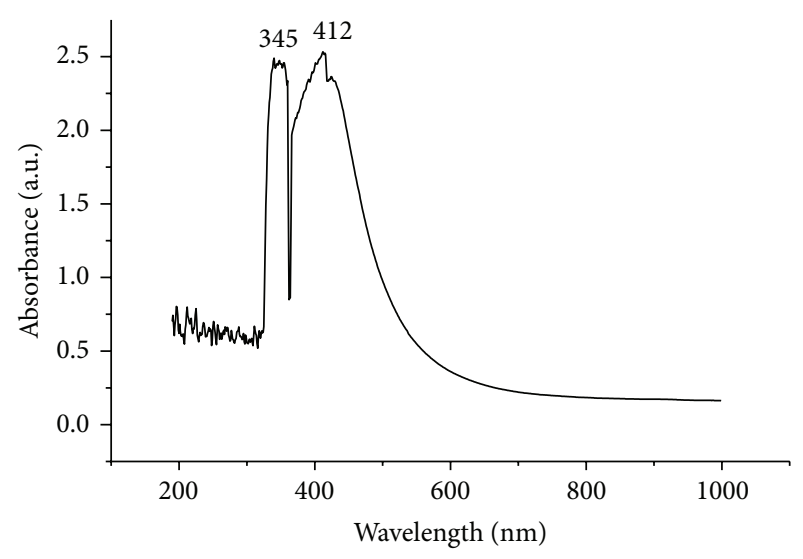

FIGURE 1: UV/visible spectrum of carbon nanoparticles.

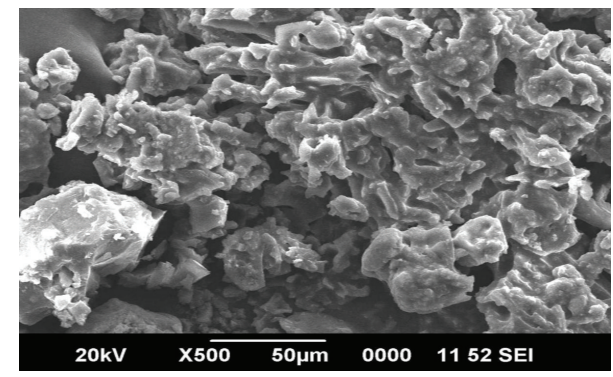

FIGURE 2: SEM image of carbon nanoparticle.

minutes. The medium was then poured into Petri dishes with almost equal agar thickness $(2.5 \mathrm{~mm})$. The dishes were cooled for sufficient time to solidify the agar medium. Miller Hinton Agar plates were launed with bacteria using sterile cotton swabs dipped in the nutrient broth culture of bacterial strains. The disc dipped in a solution of carbon nanoparticles was applied in the launed plate. The covered dishes were kept in an incubator oven at $37^{\circ} \mathrm{C}$ for $24 \mathrm{hr}$ to test. The entire handling was done inside the laminar hood in front of a flame of a spirit lamp. After the test inhibition zone diameter was measured from the clear zone of agar dish. Sterile disc impregnated with solvent as water alone was also used as control $[8,9,19]$.

\section{Results and Discussion}

The carbon nanoparticles obtained were characterized by $\mathrm{UV} /$ visible spectroscopy, X-Ray diffraction, and scanning electron microscopy. Carbon nanoparticles were dissolved in acetone-water $(3: 1)$ mixture and subjected to UV/visible analysis. The spectrum showed peaks at $345 \mathrm{~nm}$ and $412 \mathrm{~nm}$ are due to various $\pi-\pi^{*}$ transitions. UV/visible absorption spectrum of carbon nanoparticles (sample infinity) is shown in Figure 1.

Scanning electron microscopy was used for surface analysis of the carbon nanoparticles in order to investigate the morphology of the particles that the nanoparticles are irregular in shape, and the nanoparticles occurred in an 


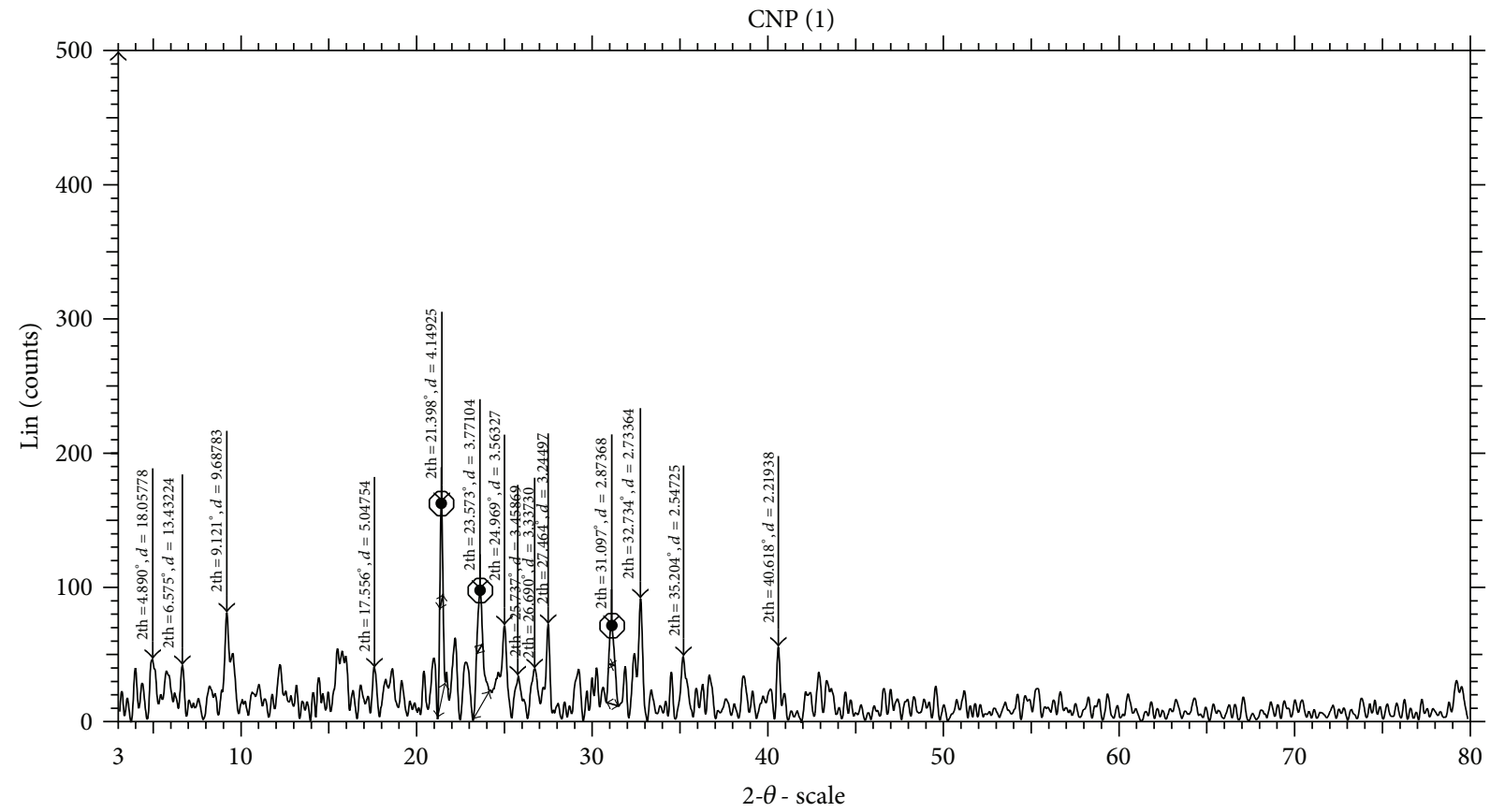

四 File: SAIFXR120616B-01(CNP (1)). Raw-step: 0.020 —-step time: $31.2 \mathrm{~s}$-WL1: 1.5406 -kA2 ratio: 0.5 - generator kV: $40 \mathrm{kV}$ generator $\mathrm{mA}: 35 \mathrm{~mA}-$ type: $2 \mathrm{Th} / \mathrm{Th}$ lo

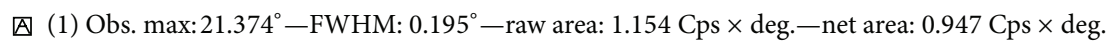

四 (2) Obs. max: $23.581^{\circ}-$ FWHM: $0.364^{\circ}-$ raw area: $1.426 \mathrm{Cps} \times$ deg. - net area: $1.092 \mathrm{Cps} \times \mathrm{deg}$.

因 (3) Obs. max: $31.087^{\circ}-$ FWHM: $0.372^{\circ}$-raw area: 0.987 Cps $\times$ deg. - net area: 0.691 Cps $\times$ deg. Operations: smooth 0.150 | background 4.571, 1.000 | background 1.000, 1.000 | smooth 0.164 | background 4.571, 0.000 | import

FIGURE 3: XRD pattern of carbon nanoparticles.

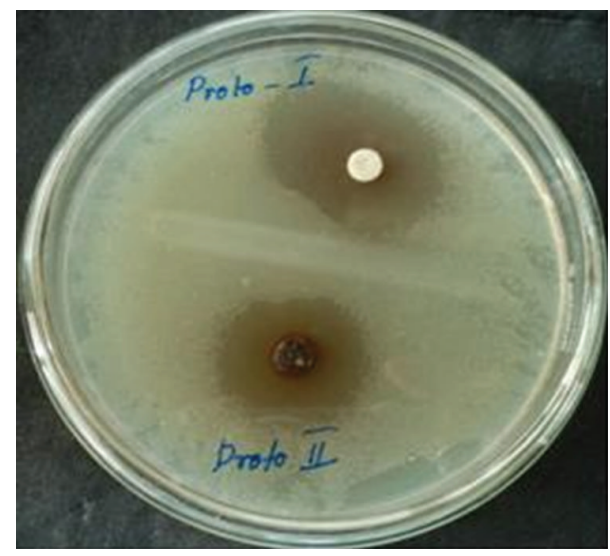

(a)

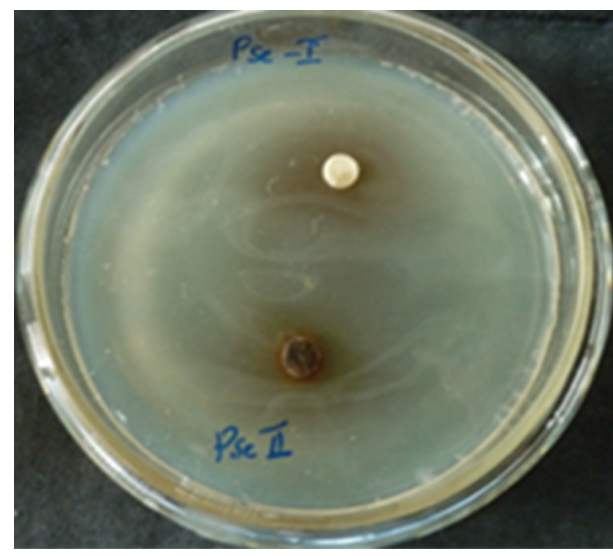

(b)

FIgURE 4: Antibacterial effects of carbon nanoparticles against (a) Proteus refrigere and (b) Pseudomonas aeruginosa.

aggregate structure. The samples were coated with a thin film of platinum to make their surface conducting. The SEM image of the carbon nanoparticle (sample infinity) is shown in Figure 2.

X-ray crystallography was used as a method of determining the arrangement of atoms within a crystal and also the size of the carbon nanoparticle. The size was found to be $20 \mathrm{~nm}-$ $40 \mathrm{~nm}$ range. The particle size was calculated using DebyeScherrer formula

$$
D=\frac{0.9 \lambda}{\beta \cos \theta},
$$




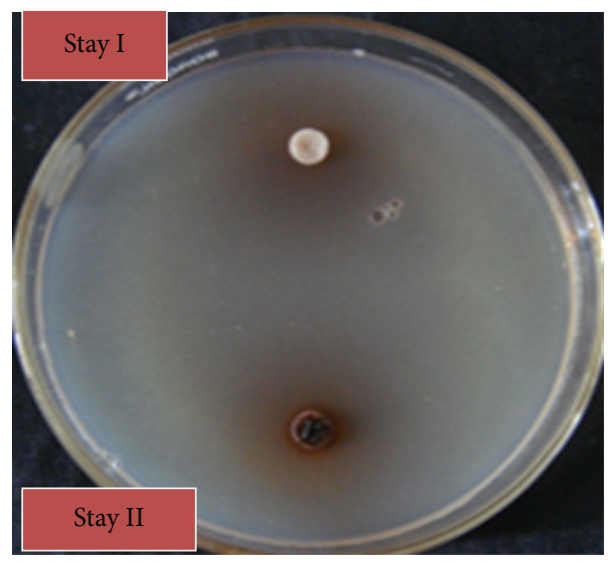

(a)

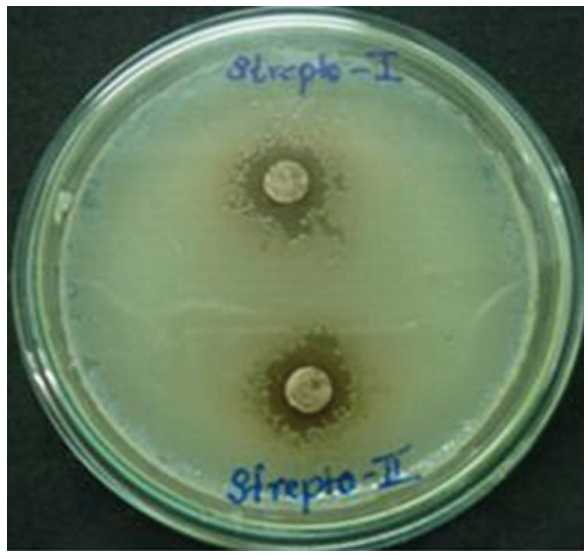

(b)

FIGURE 5: Antibacterial effects of carbon nanoparticles against (a) Staphylococcus aureus and (b) Streptococcus haemolyticus.

where $\beta$ is full width at half maximum (FWHM) which can be obtained from the XRD pattern and $\lambda$ is wavelength of X-ray which can be obtained from Bragg equation:

$$
n \lambda=2 d \sin \theta,
$$

where $n=1 ; \theta$ and $d$ can be obtained from XRD pattern [18]. The result of the X-ray diffraction (XRD) analysis of sample infinity is plotted in Figure 3.

3.1. Antibacterial Activity of Carbon Nanoparticles. The antibacterial activity of isolated carbon nanoparticle was tested by disc diffusion method.

3.2. Bacterial Strains. For in vitro screening, bacteria, such as Proteus refrigere, Pseudomonas aeruginosa, Staphylococcus aureus, and Streptococcus haemolyticus, were selected. The strains were collected and were subcultured and stocked on the semisolid nutrient Agar slants and kept at $4^{\circ} \mathrm{C}$. The micrograms were transferred to the Nutrient Broth prior to inoculation. The antibacterial activity of carbon nanoparticles isolated from carbon soot was investigated against various pathogenic bacteria of Gram-negative strains using disc diffusion method. Two samples that is, the solution of CNP isolated from acetone as sample I and the solution of CNP from acetone-water $(3: 1)$ mixture as sample II were used for the studies. Using sterile micropipette, $200 \mu$ lts of the sample solutions was poured into the discs. After incubation times the different levels of zone $(200 \mu \mathrm{L})$ were measured.

The diameter of inhibition zone for Proteus refrigere was $29 \mathrm{~mm}$ in sample I and $28 \mathrm{~mm}$ in sample II. The inhibition zone for Pseudomonas aeruginosa was found to be $22 \mathrm{~mm}$ and $20 \mathrm{~mm}$ in sample I and sample II respectively. The inhibition zone for Staphylococcus aureus was found to be $20 \mathrm{~mm}$ and $19 \mathrm{~mm}$ and that for Streptococcus haemolyticus was found to be 20 and 21 in sample I and sample II respectively (Figures 4 and 5). The nanoparticles might cross the cell membranes, penetrating into the interior of the cell and interacting with intracellular sites, by preventing bacteria from dividing and multiplying. It induces cell lysis and kills the bacteria. The inhibition zone measured in millimeter clearly demonstrated the potential activity of carbon nanoparticles against
TABLE 1: Antibacterial activity of carbon nanoparticles.

\begin{tabular}{|c|c|c|c|}
\hline \multirow[t]{2}{*}{ Sl. number } & \multirow[t]{2}{*}{ Bacterial strain tested } & \multicolumn{2}{|c|}{$\begin{array}{l}\text { Diameter of zone of } \\
\text { inhibition (in } \mathrm{mm} \text { ) }\end{array}$} \\
\hline & & Sample I & Sample II \\
\hline 1 & Proteus refrigere & 29 & 28 \\
\hline 2 & Pseudomonas aeruginosa & 22 & 20 \\
\hline 3 & Staphylococcus aureus & 20 & 19 \\
\hline 4 & Streptococcus haemolyticus & 20 & 21 \\
\hline
\end{tabular}

Gram-negative bacterial strains such as Proteus refrigere and Pseudomonas aeruginosa and Gram-positive Staphylococcus aureus and Streptococcus haemolyticus. The results are summarized in Table 1.

\section{Conclusion}

Carbon nanoparticles were isolated from natural sources such as kitchen soot and characterized by UV/visible spectroscopy, SEM, and XRD. The isolated CNPs were tested against various pathogenic bacteria. The antibacterial properties of carbon nanoparticles collected from natural sources were studied and found to be effective against bacterial strains such as Proteus refrigere, Pseudomonas aeruginosa, Staphylococcus aureus, and Streptococcus haemolyticus. The kitchen soot has been employed as an antimicrobial agent since ancient times, as the "old woman's remedy." This is nature friendly, inexpensive and can be scaled up to any quantity. The work presented in this paper can be extended further to develop a series of antimicrobial agents of natural origin and this work explores the immense possibilities in utilizing our natural resources as biomedical and therapeutic agents if these are formulated into nanosized and nanostructured materials.

\section{Acknowledgment}

The authors thank the Department of Science and Technology (Ministry of Science and Technology), Government of 
India, New Delhi, for financial support by awarding a major research project (no. SR/S1/OC-24/2006, dtd.26.10.2006).

\section{References}

[1] L. Han, D. Ghosh, W. Chen, S. Pradhan, X. Chang, and S. Chen, "Nanosized carbon particles from natural gas soot," Chemistry of Materials, vol. 21, no. 13, pp. 2803-2809, 2009.

[2] T. Pradeep, NANO: The Essentials, Tata McGraw-Hill Education, New Delhi, India, 2007.

[3] S. C. Ray, A. Saha, N. R. Jana, and R. Sarkar, "Fluorescent carbon nanoparticles: synthesis, characterization, and bioimaging application," Journal of Physical Chemistry C, vol. 113, no. 43, pp. 18546-18551, 2009.

[4] H. Chen and M. Roco, Mapping Nanotechnology Innovations and Knowledge, Global and Longitudinal Patent and Literature Analysis Series, Springer, New York, NY, USA, 2009.

[5] S. V. Kyriacou, W. J. Brownlow, and X. N. Xu, "Using nanoparticle optics assay for direct observation of the function of antimicrobial agents in single live bacterial cells," Biochemistry, vol. 43, no. 1, pp. 140-147, 2004.

[6] M. L. Abarca, M. R. Bragulat, G. Castellá, and F. J. Cabañes, "Ochratoxin A production by strains of Aspergillus niger var. niger," Applied and Environmental Microbiology, vol. 60, no. 7, pp. 2650-2652, 1994.

[7] E. Schuster, N. Dunn-Coleman, J. Frisvad, and P. van Dijck, "On the safety of Aspergillus niger-a review," Applied Microbiology and Biotechnology, vol. 59, no. 4-5, pp. 426-435, 2002.

[8] C. George, S. Kuriakose, B. Prakashkumar, and T. Mathew, "Synthesis, characterisation and antibacterial applications of water-soluble, silver nanoparticle-encapsulated $\beta$-cyclodextrin," Supramolecular Chemistry, vol. 22, no. 9, pp. 511-516, 2010.

[9] C. George, S. Kuriakose, S. George, and T. Mathew, "Antifungal activity of silver nanoparticle-encapsulated $\beta$-cyclodextrin against human opportunistic pathogens," Supramolecular Chemistry, vol. 23, no. 8, pp. 593-597, 2011.

[10] R. Muntaha, Study of the nanomaterials and their antimicrobial activities [Ph.D. thesis], Texas Southern University, 2009.

[11] S. Liu, L. Wei, L. Hao et al., "Sharper and faster "nano darts" kill more bacteria: a study of antibacterial activity of individually dispersed pristine single-walled carbon nanotube," ACS Nano, vol. 3, no. 12, pp. 3891-3902, 2009.

[12] G. Jia, H. Wang, L. Yan et al., "Cytotoxicity of carbon nanomaterials: single-wall nanotube, multi-wall nanotube, and fullerene," Environmental Science and Technology, vol. 39, no. 5, pp. 13781383, 2005.

[13] L. Dong, A. Henderson, and C. Field, "Antimicrobial activity of single-walled carbon nanotubes suspended in different surfactants," Journal of Nanotechnology, vol. 2012, Article ID 928924, 7 pages, 2012.

[14] C. Yang, J. Mamouni, Y. Tang, and L. Yang, "Antimicrobial activity of single-walled carbon nanotubes: length effect," Langmuir, vol. 26, no. 20, pp. 16013-16019, 2010.

[15] L. R. Arias and L. Yang, "Inactivation of bacterial pathogens by carbon nanotubes in suspensions," Langmuir, vol. 25, no. 5, pp. 3003-3012, 2009.

[16] S. Kang, M. Herzberg, D. F. Rodrigues, and M. Elimelech, "Antibacterial effects of carbon nanotubes: size does matter!" Langmuir, vol. 24, no. 13, pp. 6409-6413, 2008.

[17] P. Misra and M. A. Dubinsky, Ultraviolet Spectroscopy and UV Lasers, Marcel Dekker, New York, NY, USA, 2002.
[18] G. I. Goldstein, D. E. Newbury, P. Echlin, D. C. Joy, C. Fiori, and E. Lifshin, Scanning Electron Microscopy and X-Ray Microanalysis, Plenum Press, New York, NY, USA, 1981.

[19] Laboratory Procedures for Microorganisms, http://www.cabri. .org/guidelines/micro-organisms/M203Ap1.html. 

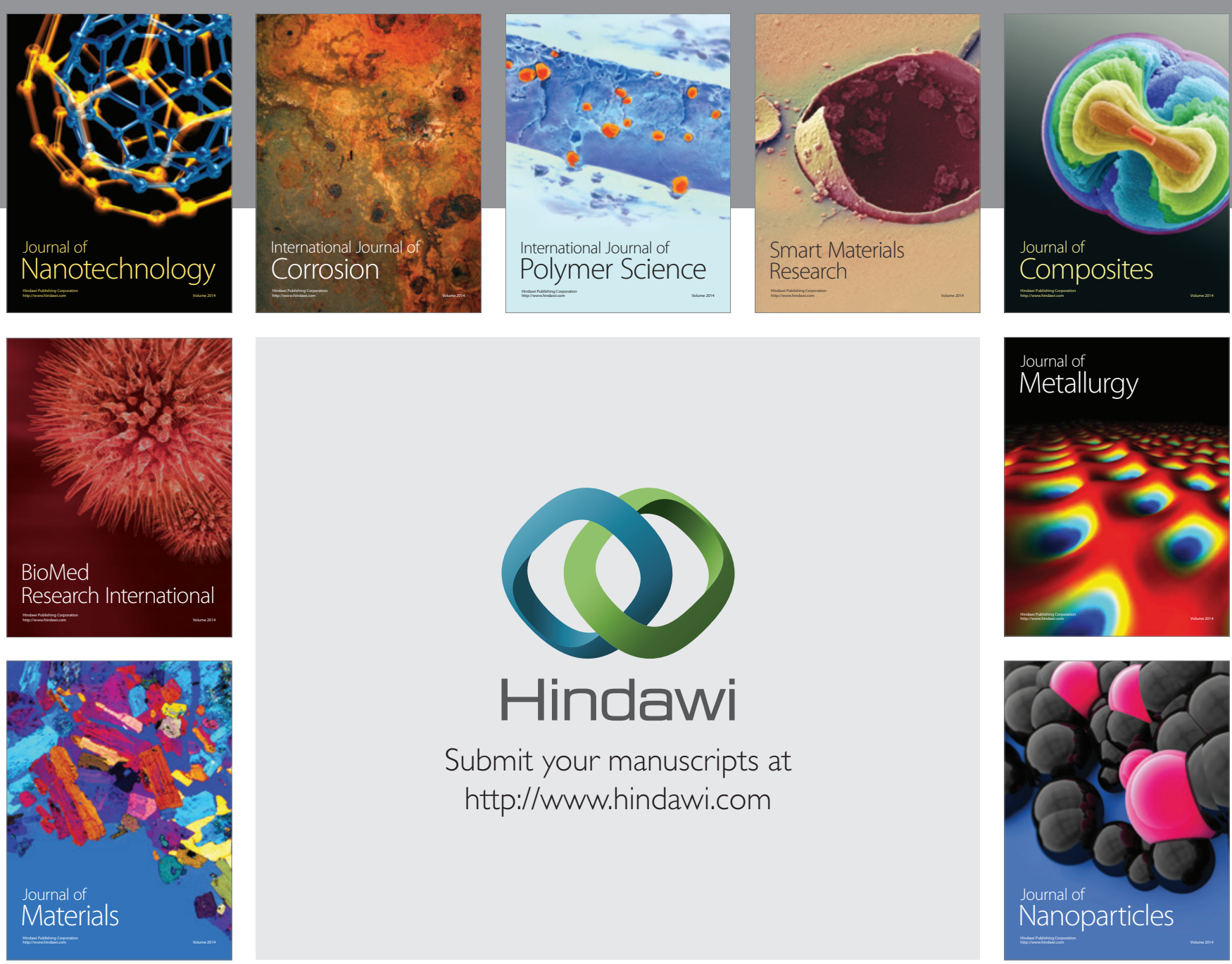

Submit your manuscripts at http://www.hindawi.com
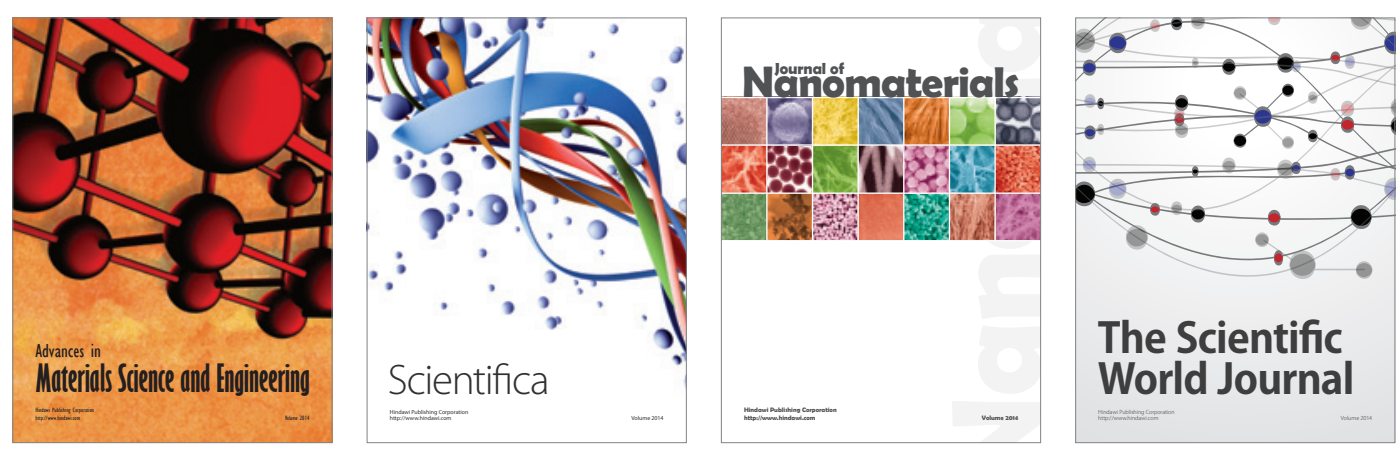

\section{The Scientific World Journal}
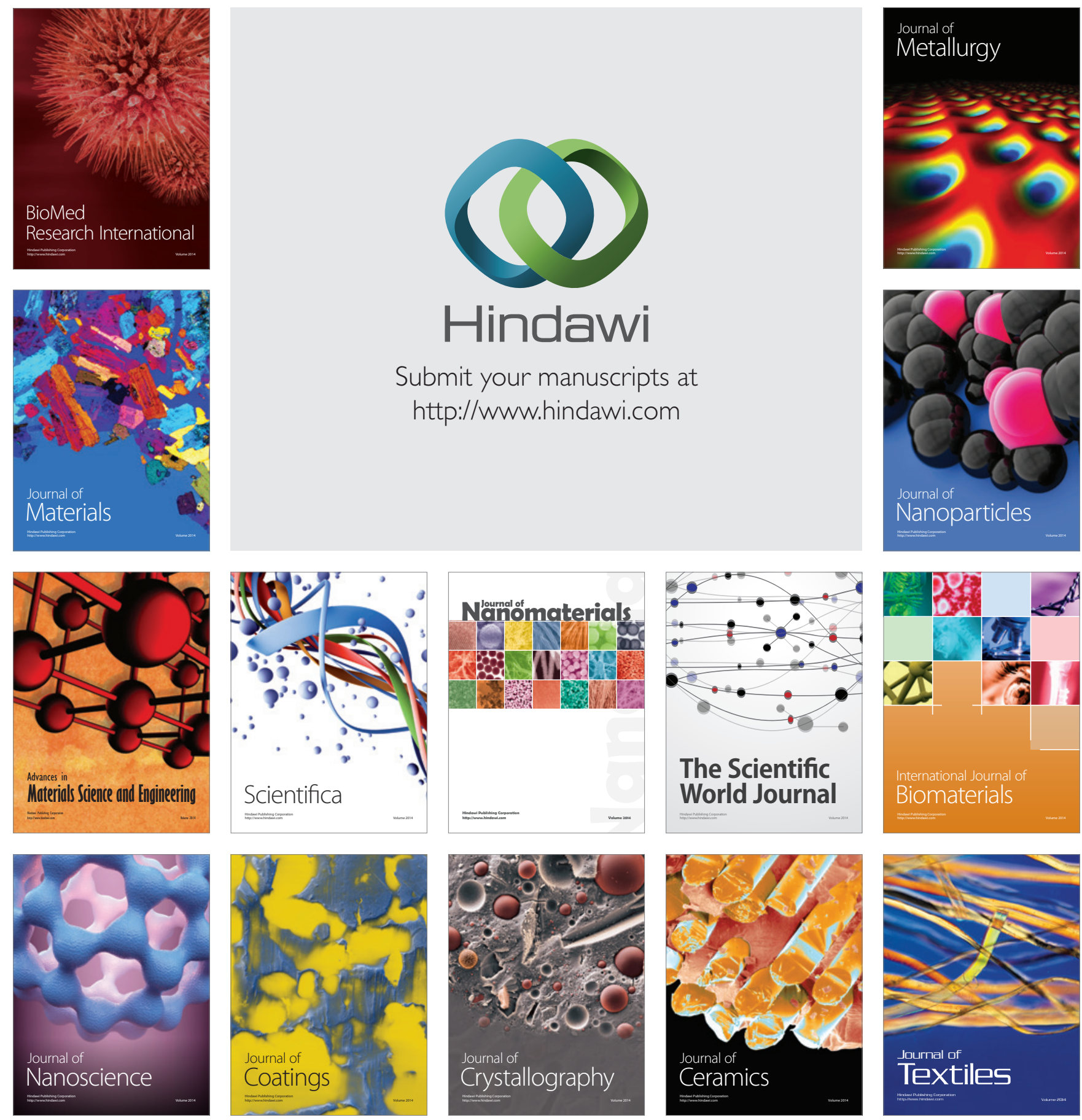